\title{
A POSITION SENSITIVE HIGH RESOLUTION HODOSCOPE FOR PARTICLE UNSTABLE INTERMEDIATE MASS FRAGMENTS
}

\author{
T. MURAKAMI, T.K. NAYAK, W.G. LYNCH, K. SWARTZ *, Z. CHEN, D.J. FIELDS **, \\ C.K. GELBKE, Y.D. KIM, M.R. MAIER, J. POCHODZALLA ${ }^{+}$, M.B. TSANG, H.M. XU \\ and F. ZHU
}

National Superconducting Cyclotron Laboratory and Department of Physics and Astronomy, Michigan State University, East Lansing, MI 48824, USA

Received 14 April 1988 and in revised form 28 July 1988

\begin{abstract}
A position sensitive high resolution hodoscope was developed to detect particle unstable intermediate mass fragments and to resolve the individual excited states of these nuclei. The hodoscope consists of 13 telescopes, four of these telescopes were designed to Isotopically resolve fragments with $3 \leqq Z \leqq 10$ and the other nine to resolve hydrogen and helium isotopes. In order to optimize the excitation energy resolution of the hodoscope, each telescope contains an $x-y$ position sensitive gas proportional counter. A position resolutıon better than $0.5 \mathrm{~mm}$ is obtained for $5.8 \mathrm{MeV} \alpha$-particles. This position sensitivity also allows compensation for the thickness nonuniformity of the nonplanar silicon detectors incorporated in the nine light particle telescopes. For proton decay channels of the particle unstable nucleus ${ }^{14} \mathrm{~N}^{*}$ produced in ${ }^{14} \mathrm{~N}$ induced reactions on ${ }^{\text {nat }} \mathrm{Ag}$ at $E / A=35 \mathrm{MeV}$, the hodoscope provides an excitation energy resolution of about $60 \mathrm{keV}$ (FWHM).
\end{abstract}

\section{Introduction}

Many properties of the fragments with $3 \leqq Z \leqq 20$, produced in energetic nuclear reactions, are determined by the conditions which prevail at "thermal freezeout" at which the probability for further interactions between the fragment and remaining system becomes negligible. If the internal excitation energy of the system is large at freezeout, many of the fragments are emitted in excited states; if the internal excitation energy is small, few fragments are excited. The relative populations of states of a given fragment therefore provide a measure of the internal excitation energy of the fragmentıng system at freezeout.

Relative populations of excited states have been measured for a variety of reactions and emitted fragments (for example refs. [1-3]). According to many statistical models, the ratio $N_{1} / N_{2}$ of the populations of two relatively narrow excited states of a fragment is given approximately by

$N_{1} / N_{2}=\left(2 J_{1}+1\right) /\left(2 J_{2}+1\right) \exp \left(-\Delta E / T_{\mathrm{em}}\right)$.

* Present address: Department of Physics, University of Washington, Seattle, WA 98195, USA.

** Present address: Physics Division, Lawrence Livermore National Laboratory, Livermore, CA 94550, USA

+ Present address: Institut für Kernphysik, Universität Frankfurt, D-6000 Frankfurt, FRG.
Here, $\Delta E=E_{1}^{*}-E_{2}^{*}, J_{1}$ and $E_{1}^{*}$ are the spin and excitation energy, respectively, of the $i$ th state of the fragment, and $T_{\mathrm{em}}$ is the "emission temperature" which characterizes the internal excitation energy of the fragmenting system at freezeout [4]. If such a thermal expression is reasonable and feeding to these excited states from the sequential decay of heavier nuclei is not significant, one may determine $T_{\mathrm{em}}$ from the populations of only two states of a fragment. A measurement involving only two states, however, does not test the internal consistency of this approach. For a detailed test, the relative populations of many ( $\gg 2$ ) states of a given fragment should be examined. This requires the study of fragments which have many well resolved excited states.

To perform this test, we have designed a hodoscope for measuring the populations of particle unstable states of intermediate mass fragments with $10 \leqq A \leqq 16$. Since the cross sections and the energies separating the excited states of these fragments are often small, the detection apparatus must have both a high efficiency and a high excitation energy resolution. Computer simulations revealed that the excitation energy resolution of the hodoscopes is limited primarily by the angular resolution of the detectors which detect the coincident daughter fragments from the particle decay of the excited nucleus. To achieve both high efficiency and resolution, we have constructed a position sensitive detection array which can be placed rather close to the target. 
An overall description of the various components of the detection array is given in the next section. Details of the construction and operation of the position sensitive gas detector elements are presented in section 3. Properties of the light particle and the heavy fragment energy loss telescopes are given in section 4. Issues concerning the determination of the efficiency and resolution of the array are discussed in section 5 and in the appendix. Section 5 gives a comparison between calculated and measured excitation energy spectra. A summary concludes the paper.

\section{Description of the detection array}

A schematic front view of the detection apparatus is shown in fig. 1. The hodoscope consists of nine light particle telescopes (LP) and four heavy fragment telescopes (HF). One light particle telescope is situated at the center of the array. The four heavy fragment telescopes are situated above, below and to the left and right of the central light particle telescope. At the periphery of the array are situated eight additional light particle telescopes. The light particle and heavy fragment telescopes have solid angles of 4.5 and $5.7 \mathrm{msr}$, respectively. The angular separation between adjacent telescopes is $8^{\circ}$.

A cross sectional view of the array including the central light particle telescope is shown in fig. 2 . The light particle and heavy fragment telescopes are located at different distances from the target. Expanded views of the two types of telescopes are shown in fig. 3. Both

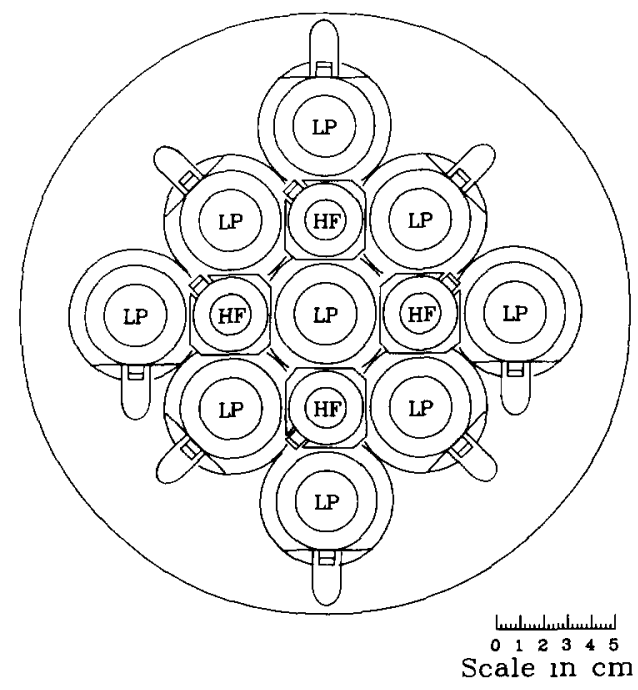

Fig 1. Front view of the hodoscope showing all the nine light particle (LP) and four heavy fragment (HF) telescopes. The actual dimensions of heavy fragment detectors are displayed. Since they are closer to the target, however, they cover larger solid angles than suggested by this projection.

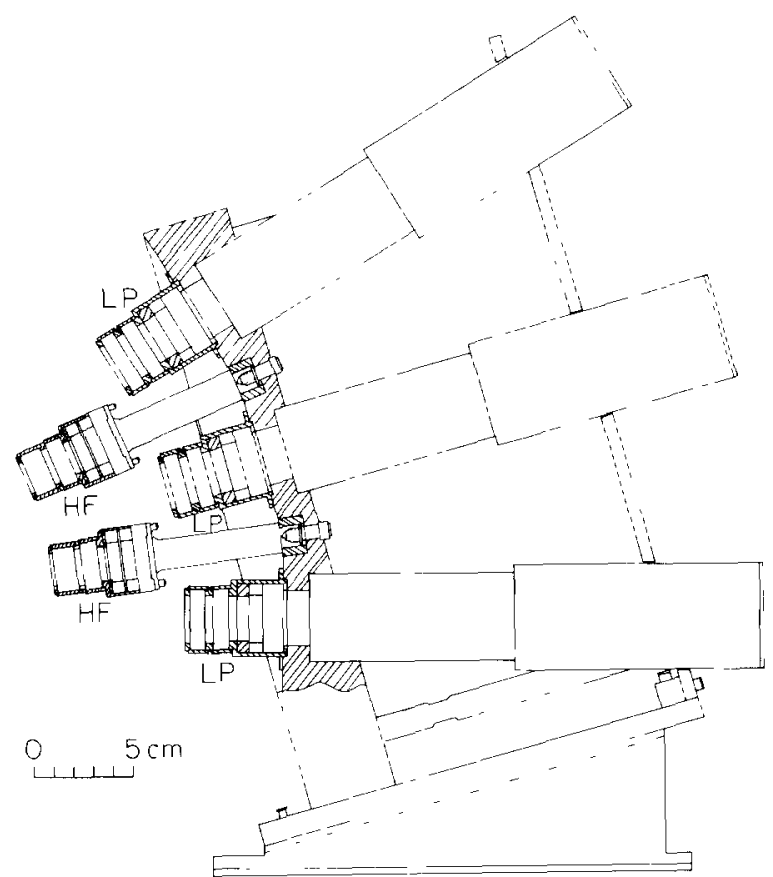

Fig. 2. Schematic cross section view of the hodoscope.

light particle and heavy fragment telescopes consist of two independent single wire proportional counters, providing position information along two orthogonal coordinates (here denoted by $x$ and $y$ ), followed by triple element energy loss telescopes; for further detail, see sections 3 and 4 . The defining apertures for the telescopes were located between the $x-y$ position sensitive proportional counters and the triple element telescopes and were situated at distances of 27.3 and $20.3 \mathrm{~cm}$ from the target for the light particle and heavy fragment telescopes, respectively. The staggering of the light particle and heavy fragment telescope allowed a maximization of the detection efficiency because it minimized the dead area between telescopes normally occupied by the detector cases and mounts.

For the detection of light particles, a nonplanar 200 $\mu \mathrm{m}$ silicon surface barrier detector of $450 \mathrm{~mm}^{2}$ surface area was used for the first element, a nonplanar $5 \mathrm{~mm}$ thick $\mathrm{Si}(\mathrm{Li})$ of $500 \mathrm{~mm}^{2}$ surface area was used for the second element and a $10 \mathrm{~cm}$ thick $\mathrm{NaI}(\mathrm{Tl})$ scintillation detector was used for the third element. The $5 \mathrm{~mm}$ $\mathrm{Si}(\mathrm{Li})$ detectors were fabricated with a total dead layer less than $15 \mu \mathrm{m}$ [5]. The silicon detectors for these telescopes were mounted on the front and the $\mathrm{NaI}(\mathrm{Tl})$ detectors on the rear of a mounting plate consisting of a spherical section subtending a half angle of $16^{\circ}$. For the detection of heavy fragments, planar $75 \mu \mathrm{m}$ and 100 $\mu \mathrm{m}$ silicon surface barrier detectors of $300 \mathrm{~mm}^{2}$ surface area and $1.5 \%$ thickness uniformity were used for the first and second elements; a $5 \mathrm{~mm}$ thick $\mathrm{Si}(\mathrm{Li})$ detector of $400 \mathrm{~mm}^{2}$ was used for the third element. The heavy 

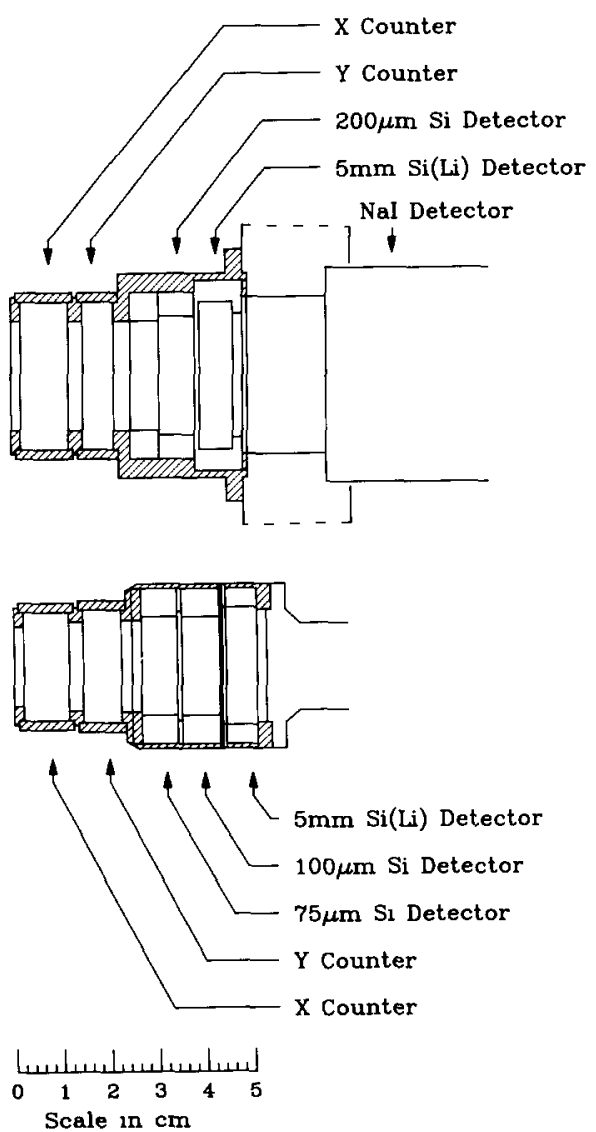

Fig. 3. Expanded drawng showing the LP (top) and HF (bottom) telescopes. fragment telescopes were positioned in front of the light particle telescopes by cylindrical rods which were bolted to the mounting plate. A photograph of the assembled detection array is shown in fig. 4. All silicon surface barrier detectors were supplied by EG\&G Ortec. The $\mathrm{Si}(\mathrm{Li})$ detectors were fabricated by Lawrence Berkeley Laboratory.

\section{Position sensitive gas proportional counters}

Position information for each individual telescope was obtained with two single wire gas proportional counters each providing one coordinate of a two-dimensional Cartesian readout. A photograph of the individual components of these counters is shown in fig. 5 . Each counter was cylindrical in shape with length of 1.2 $\mathrm{cm}$ and diameters of 3 and $2.3 \mathrm{~cm}$ for $\mathrm{LP}$ and $\mathrm{HF}$ detectors, respectively. The anode wire was situated in the middle of each counter along the circular diameter and insulated from the detector case by G-10 feedthroughs. The position along each wire was obtained by resistive charge division. This readout scheme appeared to be more linear and more space efficient than a comparable drift chamber configuration. The front and rear windows consisted of $6 \mu \mathrm{m}$ Mylar $\left(\left(\mathrm{C}_{10} \mathrm{H}_{8} \mathrm{O}_{4}\right)_{n}\right)$ aluminized on the interior to provide a cathode surface. A $1.5 \mu \mathrm{m}$ Mylar foil, aluminized on both sides, separated the $x$ and $y$ position counters. The anode wire was made by $7.6 \mu \mathrm{m}$ Nichrome wire having total resistances of approximately 600 and $400 \Omega$

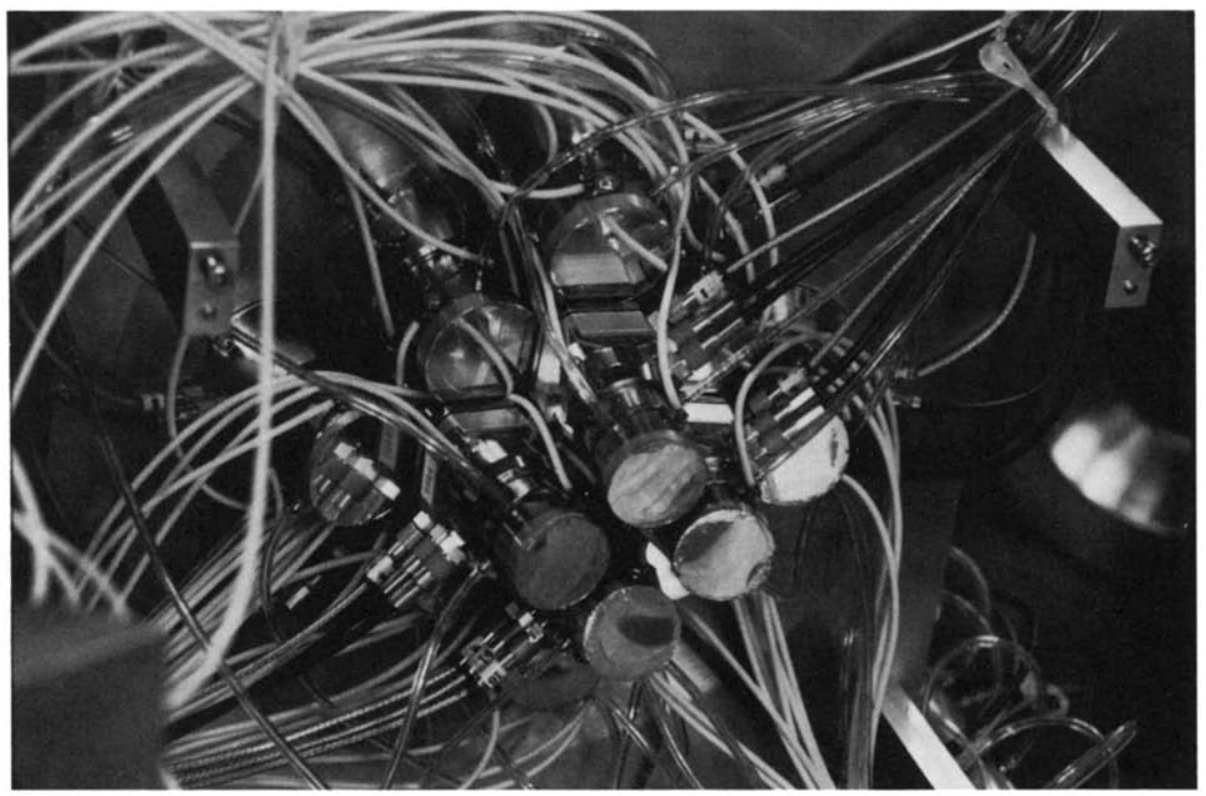

Fig. 4. Photograph of the assembled hodoscope. The four HF telescopes are in the foreground. Eight out of nine LP telescopes can be seen at a larger distance from the target. 


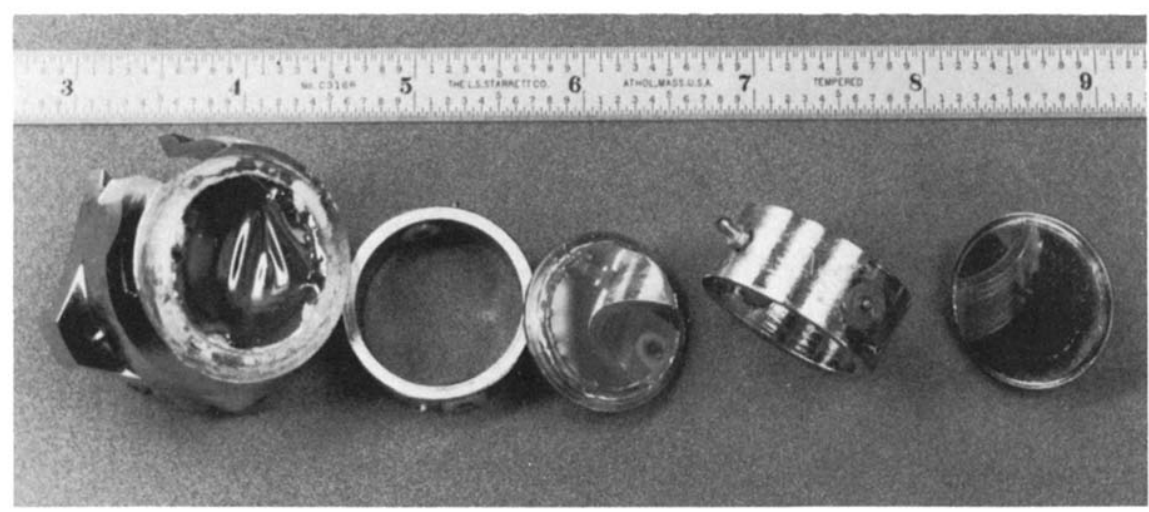

Fig. 5. Photograph showing individual components of HF position sensitive gas detector. From right to left one sees the entrance window, front cylinder, middle foil, back cylinder, and exit window which also serves as a mount for $\Delta E$ and $E$ silicon surface barrier detectors. A ruler provides the scale in inches.

for the light particle and heavy fragment telescopes, respectively.

The low resistance of the anode wires requires preamplifiers with a low input impedance and a moderately low noise figure. Since the signal to noise ratio is improved by reducing the capacitance of the cable between detector and preamplifier, a charge sensitive preamplifier was designed that could be placed in vacuum next to the detectors. Fig. 6 shows the schematic circuit diagram of the preamplifier. The circuit was designed with bipolar transistors exclusively. The input impedance, $Z_{\mathrm{tn}}$, of a charge sensitive preamplifier is given by [6]

$Z_{\mathrm{in}}=\frac{C_{0}}{g_{\mathrm{m}} C_{\mathrm{f}}}$,

where $g_{\mathrm{m}}$ is the transconductance of the input transistor (designated $\mathrm{T} 1$ in the figure), $C_{\mathrm{f}}$ is the feedback capacitor, and $C_{0}$ is the "rolloff" capacitor from the collector (or drain) to ground. In our circuit, $C_{0}=C_{\mathrm{f}}$ and the input impedance is $Z_{\mathrm{in}}=1 / g_{\mathrm{m}}=30 \Omega$. To avoid overheating in vacuum, the preamplifier was designed to have a low power dissipation of about 100 $\mathrm{mW}$. The low standing currents $(1 \mathrm{~mA})$ and the large

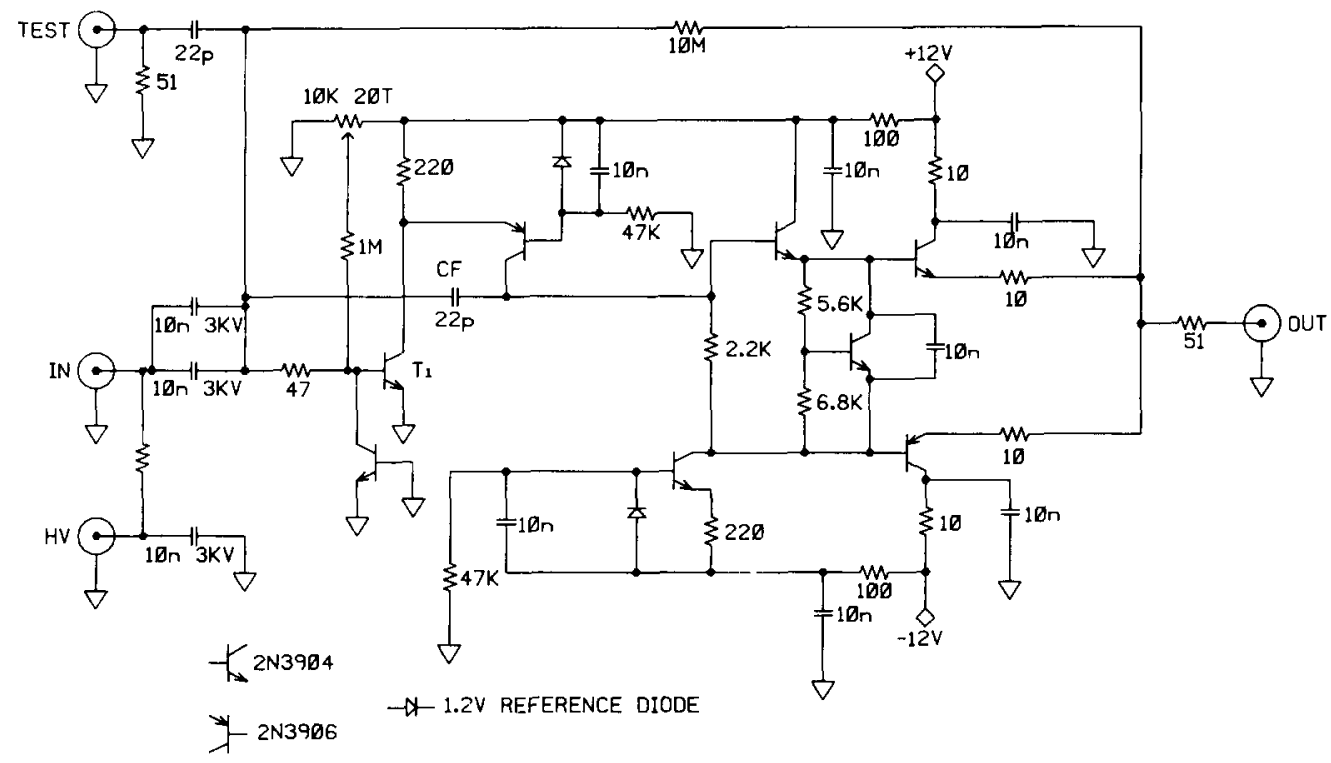

Fig. 6. Schematic circuit diagram of the low input impedance preamplifier used for the position sensitive gas detectors. 
feedback capacitor $(22 \mathrm{pF})$ limit the slew rate of this circuit to $\mathrm{SR}=I / C_{\mathrm{f}}=50 \mathrm{~V} / \mu \mathrm{s}$.

The efficiency and long term stability of the gas counters were tested with a variety of gas mixtures and pressures. Isobutane $\left(\left(\mathrm{CH}_{3}\right)_{2} \mathrm{CHCH}_{3}\right)$ offered both high efficiency and high resolution, but the performance of the gas detectors with isobutane was degraded seriously after about $4 \times 10^{8}$ counts, with the gas gain decreasing by at least a factor of 2 . Such deterioration in counter performance is caused by hydrocarbon polymerization on the electrodes $[7,8]$.

Detector lifetimes can be improved by adding nonpolymerizing quenchers, such as isopropyl alcohol $\left(\left(\mathrm{CH}_{3}\right)_{2} \mathrm{CHOH}\right)$ or methylal $\left(\mathrm{CH}_{2}\left(\mathrm{OCH}_{3}\right)_{2}\right)$, to the gas mixture [7]. We tested our detectors with different mixtures of isobutane and methylal. For practically all mixing ratios, counter had good efficiency - even for pure methylal. Better resistance to aging effects appeared at higher methylal concentrations. Since high methylal concentrations may adversely affect counter and gas handling system components [8], however, we have chosen the $20 \%$ methylal, $80 \%$ isobutane gas mixture for actual experiments.

During experiments, the heavy fragment telescope was operated at a pressure of 40 Torr and a voltage of $900 \mathrm{~V}$, while the light particle telescope was operated at 100 Torr and $1250 \mathrm{~V}$. At these pressures, the detection efficiencies for light particles and heavy fragments were $100 \%$ over the energy range of interest $(5 \mathrm{MeV} \leqq E / A$ $\leqq 40 \mathrm{MeV}$ ). These high operating voltages correspond to the upper portions of the proportional regime approaching the domain of limited proportionality. A constant gas flow rate was maintained for all telescopes such that $20 \%$ of the counter gas was replaced every minute.

The position spectra of the gas counters were calibrated with the 5.805 and $5.763 \mathrm{MeV} \alpha$-particles from a ${ }^{244} \mathrm{Cm}$ source which was placed at the target location. A calibration mask with holes of $1 \mathrm{~mm}$ diameter, separated by $1.5 \mathrm{~mm}$, was placed in front of the hodoscope at a distance of $16.5 \mathrm{~cm}$ from the target center. Because of the higher energy loss of low energy $\alpha$-particles, this calibration was performed at the lower operating voltage of $1150 \mathrm{~V}$ for the light particle telescopes. Nonlinearities of the $x-y$ position spectrum were corrected by the empirical transformations;

$$
\begin{aligned}
X= & a_{0}+a_{1} X_{\mathrm{m}}+a_{2} Y_{\mathrm{m}}+a_{3} X_{\mathrm{m}}^{2}+a_{4} X_{\mathrm{m}} Y_{\mathrm{m}}+a_{5} Y_{\mathrm{m}}^{2} \\
& +a_{6} X_{\mathrm{m}}^{3}+a_{7} X_{\mathrm{m}}^{2} Y_{\mathrm{m}}+a_{8} X_{\mathrm{m}} Y_{\mathrm{m}}^{2}+a_{9} Y_{\mathrm{m}}^{3}, \\
Y= & b_{0}+b_{1} X_{\mathrm{m}}+b_{2} Y_{\mathrm{m}}+b_{3} X_{\mathrm{m}}^{2}+b_{4} X_{\mathrm{m}} Y_{\mathrm{m}}+b_{5} Y_{\mathrm{m}}^{2} \\
& +b_{6} X_{\mathrm{m}}^{3}+b_{7} X_{\mathrm{m}}^{2} Y_{\mathrm{m}}+b_{8} X_{\mathrm{m}} Y_{\mathrm{m}}^{2}+b_{9} Y_{\mathrm{m}}^{3} .
\end{aligned}
$$

Here, $X_{\mathrm{m}}$ and $Y_{\mathrm{m}}$ denote positions directly obtained by the charge division method, and $X$ and $Y$ represent the actual positions. Coefficients $a_{t}$ and $b_{r}$ were de-

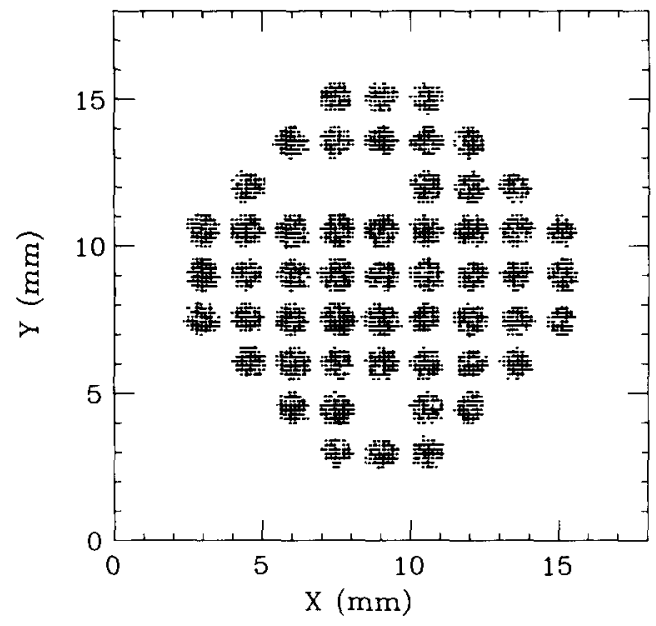

Fig 7 Two dimensional position spectrum of the calibration mask for one of the heavy fragment detectors. The missing points were used to identify and establish the onentation of the different detectors.

termined via fitting the measured mask spectrum. Fig. 7 shows the two dimensional calibration spectrum for a heavy fragment telescope after correction for nonlinearities. This spectrum and the spectra of other heavy fragment telescopes are consistent with a position resolution of $0.33 \mathrm{~mm}$ FWHM. The spectra for light particle telescopes are consistent with a slightly worse resolution of $0.50 \mathrm{~mm}$ FWHM. The position resolution was limited primarily by the preamplifier noise; it scales inversely with the signal height and therefore inversely with the energy loss in the detector gas.

The proportional counters proved to be rather sensitive to electrons and soft photons produced by the beam in the target. The corresponding background could be reduced to a tolerable level by installing $5 \mathrm{mg} / \mathrm{cm}^{2}$ $\mathrm{Au}$ foils in front of the gas detectors and adding a magnetic suppression system midway between the target and the detector array.

\section{Detector telescopes}

For ions with $E / A \geqq 5 \mathrm{MeV}$, the heavy fragment energy loss telescopes, constructed with planar silicon surface barrier detectors, provided accurate charge and mass identification via the empirical relationship [9],

$R \propto E^{b} / M q_{\mathrm{eff}}^{2}$,

where $E, R, M$ and $q_{\mathrm{eff}}$ denote the energy, range, mass and effective charge state of the fragment, respectively, and $b$ is an adjustable constant with a typical value of 
about 1.7. For a planar $\Delta E$ detector of thickness $T$ and a stopping $E$ detector, one obtains from eq. (4)

$M q_{\mathrm{eff}}^{2} \propto\left((E+\Delta E)^{b}-E^{b}\right) / T$.

Following ref. [10] we have adopted a number of empirical improvements. Particle identification is obtained, instead, using

$$
\begin{aligned}
\mathrm{PID}= & \ln \left(M q_{\mathrm{eff}}^{2}\right) \\
= & \ln (b \Delta E)+(b-1) \ln (E+c \Delta E) \\
& -b \ln (300),
\end{aligned}
$$

$b=1.825-0.18 \frac{\Delta E[\mathrm{MeV}]}{T[\mu \mathrm{m}]}, \quad c=0.5$.

Fig. 8 shows the particle identification achieved for particles which stop in the second $(100 \mu \mathrm{m})$ element of the HF telescope. Isotopic resolution is achieved for all elements displayed. Similarly, good resolution is obtained for heavy fragments which stop in the third element $\left(5 \mathrm{~mm} \mathrm{Si}\left(\mathrm{L}_{1}\right)\right)$ of the telescope.

Because of cost-efficiency reasons, nonplanar fully depleted detectors of $200 \mu \mathrm{m}$ thickness were used as first elements of the light particle telescopes. The detectors were fabricated by a technique producing convex shape $\mathrm{Si}$ wafers with nonuniformities of up to $25 \%$. If the variation of detector thickness is a function only of the radial distance from the center of detector $\rho(\mathrm{mm})$, we can replace $T$ in eq. (5) by

$T(\rho)=T_{0} f(\rho), \quad(f(\rho) \leqq 1)$,

where $T_{0}$ is the thickness of the detector at the center. Good particle identification can still be achieved by correcting for the thickness varnation using the position

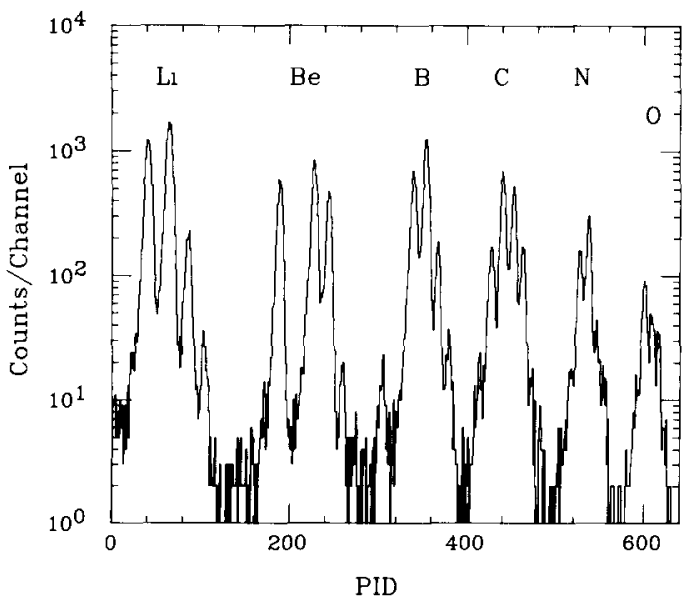

Fig. 8. Sample particle identification spectrum for a heavy fragment telescope.

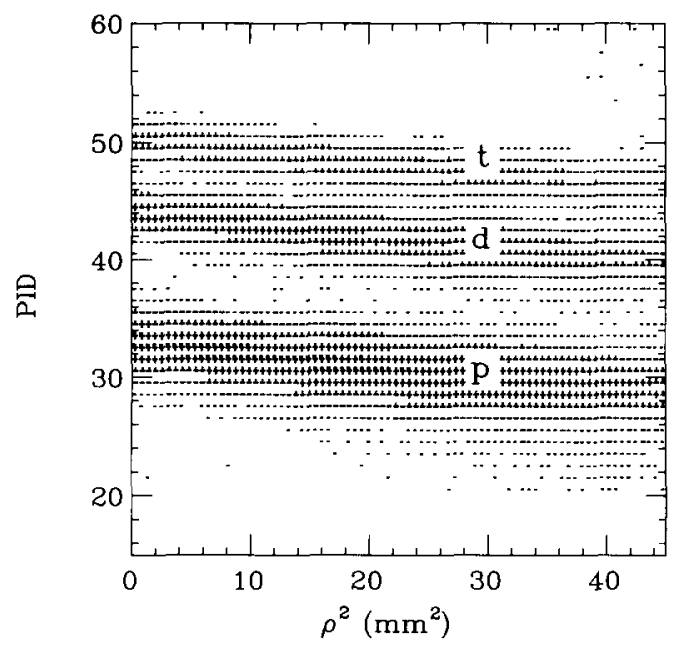

Fig. 9 Partıcle identification in the central regıon of a light particle telescope as a function of $\rho^{2}$, where $\rho$ is the radial distance from the center of the detector.

information provided by the gas detectors. With this modification eq. (6) becomes

$$
\begin{aligned}
& \mathrm{PID}=\ln (b \Delta E)+(b-1) \ln (E+c \Delta E)-b \ln (300) \\
& \quad-\ln (f(\rho)), \\
& b=1.825-0.18 \frac{\Delta E[\mathrm{MeV}]}{T_{0}[\mu \mathrm{m}]}, \quad c=0.65 .
\end{aligned}
$$

Fig. 9 shows the particle identification in the central

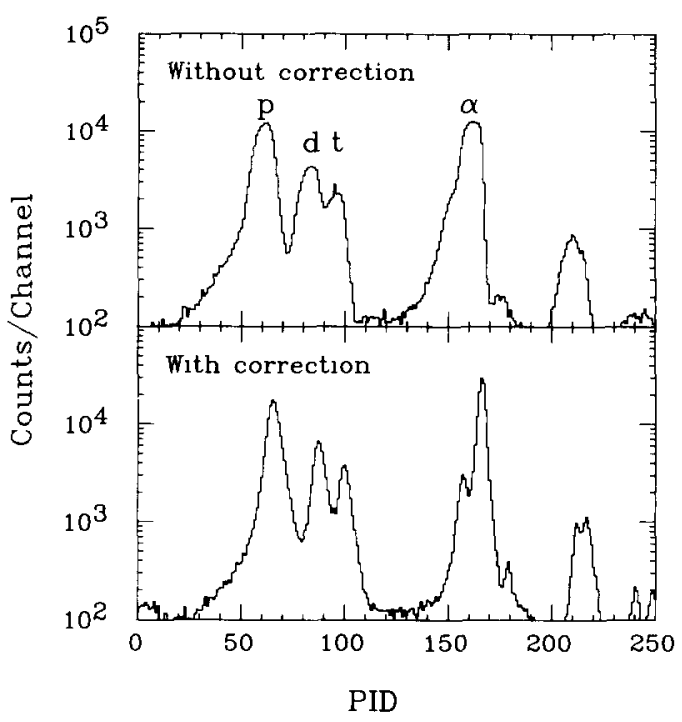

Fig. 10. Upper part: particle identıfication spectrum without correction. Lower part particle identification spectrum for a light partıcle telescope after correctıng for nonunıformity of $\Delta E$ detector. 
region of a typical detector as a function of $\rho^{2}$, obtained with $f(\rho)=1$. The PID values decrease linearly with $\rho^{2}$ by $28 \%$ from the center to the periphery of the active area. All the first elements of the nine light particle telescopes displayed similar thickness varnations ranging from $25 \%$ to $30 \%$. We adopted the functional form $f(\rho)=\exp \left(-\lambda \rho^{2}\right)$ with $\lambda=5.3 \times 10^{-3} \mathrm{~mm}^{-2}$, and improved the particle identification in fig. 10 . Clean isotope resolution was obtained for elements as heavy as lithium.

Computer simulations, described in the appendix, indicate that accurate energy calibrations of the detector telescopes are much more critical than good energy resolutions for achieving an optimal excitation energy resolution. The overall energy resolution of the silicon telescopes was about $150 \mathrm{keV}$. Before and after the experiment the detectors were calibrated at low energies with ${ }^{241} \mathrm{Am}$ and ${ }^{212} \mathrm{Po} \alpha$ sources. These calibrations were extrapolated to energies of several hundred $\mathrm{MeV}$ by injecting a signal from a precision BNC pulser into the input stage of preamplifiers. In this fashion, the relative calibrations of all silicon detectors were established to an estimated accuracy of about $0.5 \%$. Dead layers of the silicon detectors were measured with an ${ }^{241} \mathrm{Am}$ source by rotating the detectors with respect to the direction of the incident $\alpha$ particles. Calculations indicate that an error in the relative calibration of light particle and heavy fragment telescopes of about $0.5 \%$ deteriorates the excitation energy resolution as much as $5 \%$ for an excited state with excitation energy of 0.7 $\mathrm{MeV}$. A $0.5 \%$ miscalibration of the energy scale which is in the same direction for both light particle and heavy fragment telescopes does not broaden the measured states significantly; it results, instead, in a similar and less deleterious overall miscalibration of the excitation energy scale. The energies of both light particle and heavy fragment were corrected for the energies lost in the target, gold absorber, and gas counter using an energy loss lookup table based upon ref. [11].

Most light particles originating from the decay of nuclei with $10 \leqq Z \leqq 16$ were stopped in the second ( 5 $\mathrm{mm}$ ) element of the light particle telescope. Light particles from the decay of lighter nuclei $(A \leqq 9)$ frequently penetrated the $5 \mathrm{~mm}$ detector and stopped in the $\mathrm{NaI}(\mathrm{TI})$ detector. The NaI(T1) detectors have energy resolutions of about $2 \%$, adequate to resolve the states of the lighter nuclei. Calibrations for the NaI(T1) detectors were obtained by converting the $\Delta E$ information from the 5 $\mathrm{mm} \mathrm{Si}(\mathrm{Li})$ detectors to corresponding energies. These calibrations were confirmed by measuring the energies of recoil protons backscattered from a polypropylene target by a $490 \mathrm{MeV}{ }^{14} \mathrm{~N}$ beam. The energy calibrations of $\mathrm{NaI}(\mathrm{T} 1)$ detectors are estimated to be accurate to within 5\%. Gain shifts of the NaI(T1) detectors were corrected for using $\Delta E$ information from the $\mathrm{Si}(\mathrm{Li})$ detector as discussed in ref. [2].

\section{Detection efficiency and resolution}

Products from the decay of particle unstable nuclei are detected as coincident events. The energy of the coincident particles can be easily combined to obtain the relative energy and, by accumulating all the measured events, the relative energy spectrum $Y_{\text {tot }}\left(E_{\text {mea }}^{*}\right)$. The portion of the total yield of particles that proceeds through the decay of particle unstable nuclei $Y_{c}\left(E_{\mathrm{mea}}^{*}\right)$ can be related to the normalized excitation energy spectrum in the rest frame of the unstable fragment for decay into channel $c,\left[\mathrm{~d} n\left(E^{*}\right) / \mathrm{d} E^{*}\right]_{\text {, }}$, by the relation

$Y_{c}\left(E_{\text {mea }}^{*}\right)=\int \mathrm{d} E^{*} \epsilon\left(E^{*}, E_{\text {med }}^{*}\right)\left[\frac{\mathrm{d} n\left(E^{*}\right)}{\mathrm{d} E^{*}}\right]$,

where $\epsilon\left(E^{*}, E_{\text {mea }}^{*}\right)$ is called the efficiency function; $E^{*}$ and $E_{\text {mea }}^{*}$ denote the actual and measured excitation energies, respectively. Details for calculating thıs function are given in the appendix. It is affected by multiple scattering and energy loss in the target, resolution and geometry of the detector elements of the hodoscope, the energy spectra and angular distributions of the particle unstable primary fragment, and any anisotropy which may exist in decay distribution of the primary fragment in its rest frame.

Calculations were performed for the decay channel ${ }^{14} \mathrm{~N} \rightarrow{ }^{13} \mathrm{C}+\mathrm{p}$ for ${ }^{14} \mathrm{~N}$ induced reactions on ${ }^{\text {nat }} \mathrm{Ag}$ at $E / A=35 \mathrm{MeV}$. In the calculations, the energy spectra and angular distributions for particle unstable ${ }^{14} \mathrm{~N}$ nuclei were assumed to be the same as those measured for stable ${ }^{14} \mathrm{~N}$ nuclei, and the decay distribution was assumed to be isotropic in the rest frame of the excited ${ }^{14} \mathrm{~N}$ nucleus. The geometry and resolution of the hodoscope elements, and target and detector foil thicknesses were taken from conditions which prevailed during a recent experiment.

Results of calculations for the total efficiency

$\epsilon_{\text {tot }}\left(E^{*}\right)=\int \mathrm{d} E_{\text {mea }}^{*} \epsilon\left(E^{*}, E_{\text {mea }}^{*}\right)$

and the root mean square resolution

$\sigma_{E^{*}}^{\text {tot }}=\left\{\int \mathrm{d} E_{\text {mea }}^{*} \epsilon\left(E^{*}, E_{\text {mea }}^{*}\right)\left(E_{\text {mea }}^{*}-E^{*}\right)^{2}\right\}^{1 / 2}$

are shown in fig. 11. The total efficiency (shown in upper part of the figure) is normalized to 1 at the relative kinetic energy of $0.42 \mathrm{MeV}$, which corresponds to the $2^{-}\left(E^{*}=7.97 \mathrm{MeV}\right)$ excited state in ${ }^{14} \mathrm{~N}$. The resolution shown in fig. 11 is mainly limited by the position resolution of the individual telescopes.

In an actual experiment, one measures a total coincidence yield, $Y_{\text {tot }}\left(E_{\text {mea }}^{*}\right)$, which is a sum of the decay yield, $Y_{c}\left(E_{\text {mea }}^{*}\right)$, and background yield, $Y_{\text {back }}\left(E_{\text {mea }}^{*}\right)$. The background yield is due to conncidences which do 


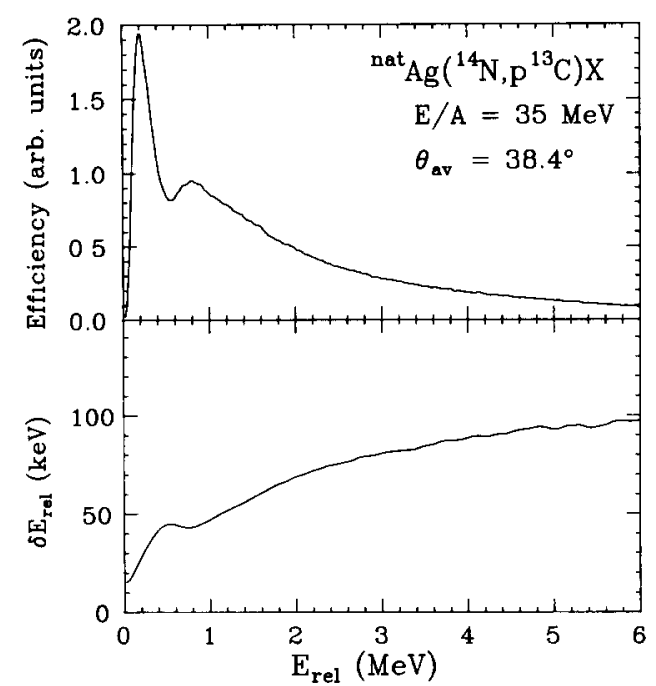

Fig. 11. Calculated total efficiency (upper part) and rms resolution (lower part) for the detection of $\mathrm{p}-{ }^{13} \mathrm{C}$ pairs resulting from the decay of particle unstable ${ }^{14} \mathrm{~N}$. The efficiency has been normalized to 1 at $E_{\mathrm{rel}}=0.42 \mathrm{MeV}\left(E^{*}=7.97 \mathrm{MeV}\right)$.

not proceed through particle unstable states. We approximate it by

$Y_{\text {back }}\left(p_{\mathrm{H}}, \boldsymbol{p}_{\mathrm{L}}\right)=C \sigma_{\mathrm{L}}\left(p_{\mathrm{L}}\right) \sigma_{\mathrm{H}}\left(p_{\mathrm{H}}\right)\left(1+R_{\text {back }}(\Delta p)\right)$,

where $C$ is a normalization constant, $\sigma_{\mathrm{L}}\left(\boldsymbol{p}_{\mathrm{L}}\right)$ and $\sigma_{H}\left(p_{H}\right)$ are the inclusive cross sections for light particles and heavy fragments, $\Delta p$ is the relative momentum between the light particle and the heavy fragment, and $\left[1+R_{\text {back }}(\Delta p)\right]$ is the background correlation function. The background correlation function is assumed to vanish for $\Delta p \rightarrow 0$ and to go to unity at large $\Delta p$ where final state interactions can be neglected. The accuracy of this approximation can be easily assessed by constructing the total correlation function, $[1+$ $R_{\text {tot }}(\Delta p)$ ], defined by

$$
Y_{\text {tot }}\left(p_{\mathrm{H}}, p_{\mathrm{L}}\right)=C \sigma_{\mathrm{L}}\left(p_{\mathrm{L}}\right) \sigma_{\mathrm{H}}\left(p_{\mathrm{H}}\right)\left(1+R_{\mathrm{tot}}(\Delta p)\right) \text {, }
$$

and investigating the correlation function at relative momenta for which no particle unstable states exist. The experimental correlation function $\left[1+R_{\text {tot }}(\Delta p)\right]$ is obtained by summing both sides of eq. (11) over all values of $p_{\mathrm{L}}$ and $\boldsymbol{p}_{\mathrm{H}}$ corresponding to a fixed relative momentum $\Delta p$ and choosing $C$ such that the total correlation function is unity at large relative momenta.

As an example, fig. 12 shows the experimental total $\mathrm{p}+{ }^{13} \mathrm{C}$ correlation function for ${ }^{14} \mathrm{~N}$ induced reactions on ${ }^{\text {nat }} \mathrm{Ag}$ at $E / A=35 \mathrm{MeV}$. Between, $7.55 \leqq E^{*} \leqq 10.27$ $\mathrm{MeV}, 16$ states should decay only by proton emission. The distinct structures observed at $E^{*}=7.97,8.49$, $\sim 9.0, \sim 9.4$ and $-10.1 \mathrm{MeV}$ correspond to groups of

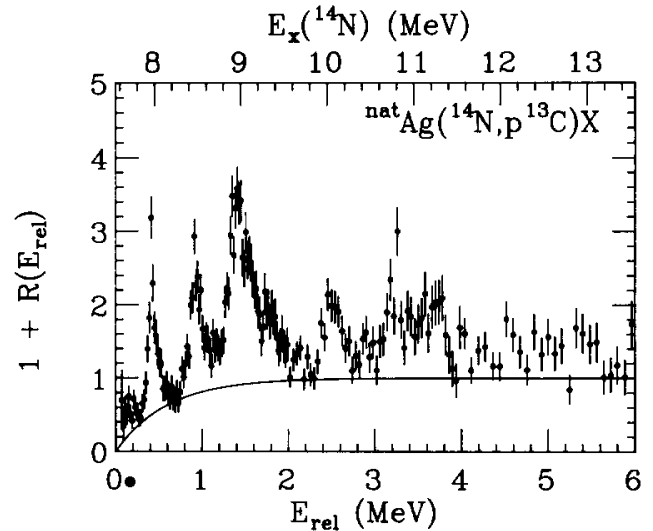

Fig. 12. $\mathrm{p}-{ }^{13} \mathrm{C}$ correlation function. The excitation energy in the ${ }^{14} \mathrm{~N}$ nucleus is indicated on the top. Solid curve indicates an estimated background.

excited states with $J \geqq 2$; additional states in this region with $J=0$ are not strongly populated. Consistent with eq. (10), the correlation function is very close to unity between the peaks and at large relative momenta where the background correlation is dominant. It also decreases to zero for small $\Delta p$. From the shape of the correlation between peaks at low relative momentum, $R_{\text {back }}(\Delta p)$ may be determined and the background yield can be subtracted from the total y1eld. Details of the calculation of the correlation function and the background are discussed in the appendix.

The resultant experimental yield is shown in fig. 13 . The rms width of the peak at $7.97 \mathrm{MeV}$ is estimated to be $48 \mathrm{keV}$, in good agreement with the calculated resolution, $45 \mathrm{keV}$. For this narrow state $\left(\Gamma_{\mathrm{cm}}=2.5 \mathrm{eV}\right)$ the observed width is governed by the instrumental resolution. It should be noted that the line shape of the peak has a longer tail than a corresponding Gaussian peak. Therefore the FWHM is significantly smaller than 2.35

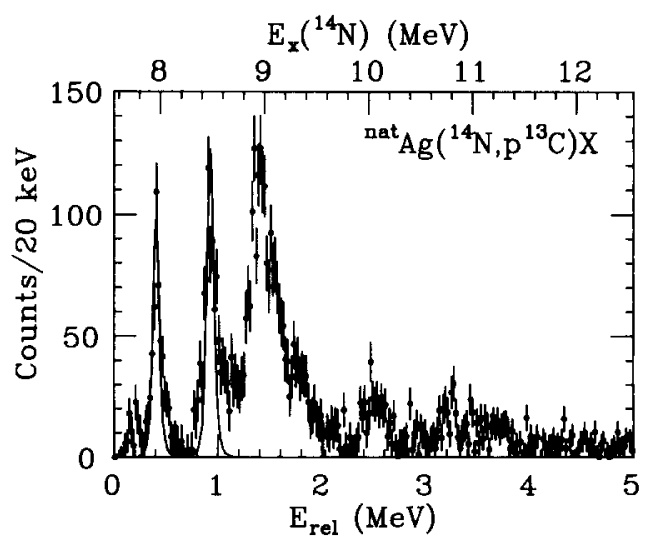

Fig. 13. Energy spectrum resulting from the decay of particle unstable ${ }^{14} \mathrm{~N}$. Solid curve is a fit described in the text. 
tumes the rms resolution given in fig. 11. In fact the FWHM of the peak at $7.97 \mathrm{MeV}$ was about $60 \mathrm{keV}$.

Experimental populations of the excited states can be extracted by comparing the experimental yield with the predictions of eq. (9). For the excitation energy spectrum, we assumed

$\left[\frac{\mathrm{d} n\left(E^{*}\right)}{\mathrm{d} E^{*}}\right]_{c}=\sum_{t} n_{t} \frac{\left(2 J_{t}+1\right) \Gamma_{t} / 2 \pi}{\left(E^{*}-E_{t}^{*}\right)^{2}+\Gamma_{t}^{2} / 4} \frac{\Gamma_{c, l}}{\Gamma_{t}}$,

where $\Gamma_{c, d} / \Gamma_{1}$ denotes the branching ratio for the decay into the channel $c$ and $n_{\imath}$, represents the population probability of the $t$ th state. A complete decomposition of the spectrum in fig. 13 is beyond the scope of the present paper. We fitted only the first two peaks corresponding to $T=0$ states in ${ }^{14} \mathrm{~N}$ at $7.97 \mathrm{MeV}\left(J^{\pi}=2^{+}\right.$, $\left.\Gamma=2.5 \times 10^{-3} \mathrm{keV}, \Gamma_{\mathrm{p}} / \Gamma_{\mathrm{tot}}=1.00\right)$ and at $8.49 \mathrm{MeV}$ ( $\left.J^{\pi}=4^{-}, \Gamma=3.5 \times 10^{-5} \mathrm{keV}, \Gamma_{\mathrm{p}} / \Gamma_{\mathrm{tot}}=0.73\right)$ [12]. The solid curve represents the calculated experimental yield corresponding to a relative population $n(7.97) / n(8.49)$ $=0.83 \pm 0.07$. The complete analysis which includes the other excited states plus decays which populate excited states of ${ }^{13} \mathrm{C}^{*}$ will be discussed in a forthcoming paper [13].

\section{Summary}

We have developed a position sensitive high resolution hodoscope for detectıng particle unstable intermediate mass fragments and numerical techniques for modelıng its efficiency and resolution. The calculation indicates that the observed experimental excitation energy resolution $\approx 60 \mathrm{keV}$ (FWHM) is limited mainly by the position resolution of the gas proportional counters. This resolution is sufficient to resolve many of the particle unstable states of p-shell nuclel and the efficiency is large enough to measure them as fragmentation products. With many states avallable for investıgation in each p-shell nucleus, more extensive tests of statistical models for fragmentation are now possible.

We have used the position information provided by the proportional counters to map out the nonuniformities $(-25-30 \%)$ of nonplanar $\Delta E$ detectors and thereby obtained mass resolution up to ${ }^{7} \mathrm{Li}$.

\section{Appendix}

\section{Detals of the efficiency calculations}

The efficiency functions $\epsilon\left(E^{*}, E_{\text {mea }}^{*}\right)$ are usually obtained by performing Monte Carlo simulations for the emission and decay of the respective particle unstable nuclei. For most detection geometries, such simulations are very time consuming because of low detection efficiencies.
We have avoided the inherent inefficiencies of such Monte Carlo simulations by calculating the efficiency function through direct integration of the two particle coincidence cross section over the detector geometry. For simplicity we assume the decay to be isotropic in the rest frame of the particle unstable nucleus and the laboratory production cross section of the particle unstable nucleus to be independent of the excitation energy $E^{*}$. The laboratory two particle concidence cross section can then be given in terms of the center of mass excitation energy spectrum and the "common" laboratory production cross section for the particle unstable nucleus $\mathrm{d} \sigma\left(E_{\mathrm{tot}}, \Omega_{\mathrm{tot}}\right) / \mathrm{d} E_{\mathrm{tot}} \mathrm{d} \Omega_{\mathrm{tot}}$ by

$$
\begin{aligned}
& \frac{\mathrm{d} \sigma\left(E_{\mathrm{H}}, \Omega_{\mathrm{H}}, E_{\mathrm{L}}, \Omega_{\mathrm{L}}\right)}{\mathrm{d} E_{\mathrm{H}} \mathrm{d} \Omega_{\mathrm{H}} \mathrm{d} E_{\mathrm{L},} \mathrm{d} \Omega_{\mathrm{L}}} \\
& =\frac{\partial\left(E_{\mathrm{rel}}, \Omega_{\mathrm{cm}}, E_{\mathrm{tot}}, \Omega_{\mathrm{tot}}\right)}{\partial\left(E_{\mathrm{H}}, \Omega_{\mathrm{H}}, E_{\mathrm{L}}, \Omega_{\mathrm{L}}\right)} \frac{\mathrm{d} \sigma\left(E_{\mathrm{tot}}, \Omega_{\mathrm{tot}}\right)}{\mathrm{d} E_{\mathrm{tot}} \mathrm{d} \Omega_{\mathrm{tot}}} \\
& \quad \times \frac{1}{4 \pi} \frac{\mathrm{d} n\left(E^{*}\right)}{\mathrm{d} E^{*}},
\end{aligned}
$$

where $\partial\left(E_{\text {rel }}, \Omega_{\mathrm{cm}}, E_{\text {tot }}, \Omega_{\text {tot }}\right) / \partial\left(E_{\mathrm{H}}, \Omega_{\mathrm{H}}, E_{\mathrm{L}}, \Omega_{\mathrm{L}}\right)$ is the Jacobian for the transformation from the center of mass coordinates $E_{\text {rel }}, \Omega_{\mathrm{cm}}$ and the laboratory coordinates $E_{\mathrm{tot}}, \Omega_{\text {tot }}$ of the parent particle unstable nucleus to the laboratory coordınates for the detected heavy $(\mathrm{H})$ and light (L) decay products. Thıs Jacobian is given by

$$
\begin{aligned}
& \frac{\partial\left(E_{\mathrm{rel}}, \Omega_{\mathrm{cm}}, E_{\mathrm{tot}}, \Omega_{\mathrm{tot}}\right)}{\partial\left(E_{\mathrm{H}}, \Omega_{\mathrm{H}}, E_{\mathrm{L}}, \Omega_{\mathrm{L}}\right)} \\
& =\frac{p_{\mathrm{L}} p_{\mathrm{H}}\left(E_{\mathrm{L}}+m_{\mathrm{L}}\right)\left(E_{\mathrm{H}}+m_{\mathrm{H}}\right)}{p_{\mathrm{L}}^{\mathrm{cm}} p_{\mathrm{tot}}\left(E_{\mathrm{L}}^{\mathrm{cm}}+m_{\mathrm{L}}\right)\left(E_{\mathrm{H}}^{\mathrm{cm}}+m_{\mathrm{H}}\right)} \\
& \quad \times\left[1-\left\{\left\{\left(p_{\mathrm{L}}^{2}+\boldsymbol{p}_{\mathrm{L}} \cdot \boldsymbol{p}_{\mathrm{H}}\right)\left(E_{\mathrm{H}}+m_{\mathrm{H}}\right)\right.\right.\right. \\
& \left.\left.\quad-\left(p_{\mathrm{H}}^{2}+\boldsymbol{p}_{\mathrm{L}} \cdot p_{\mathrm{H}}\right)\left(E_{\mathrm{L}}+m_{\mathrm{L}}\right)\right\}^{2}\right\} \\
& \quad \times\left\{\left(E_{\mathrm{L}}+E_{\mathrm{H}}+m_{\mathrm{L}}+m_{\mathrm{H}}\right)^{2}\left(E_{\mathrm{L}}+m_{\mathrm{H}}\right)\right. \\
& \left.\quad \times\left(E_{\mathrm{H}}+m_{\mathrm{H}}\right)\left(E_{\mathrm{rel}}+m_{\mathrm{L}}+m_{\mathrm{H}}\right)^{2}\right\}^{-1} \\
& \quad-\left\{\left(p_{\mathrm{L}}^{2}+\boldsymbol{p}_{\mathrm{L}} \cdot \boldsymbol{p}_{\mathrm{H}}\right)\left(E_{\mathrm{H}}+m_{\mathrm{H}}\right)^{2}\right. \\
& \left.\quad+\left(p_{\mathrm{H}}^{2}+\boldsymbol{p}_{\mathrm{L}} \cdot p_{\mathrm{H}}\right)\left(E_{\mathrm{L}}+m_{\mathrm{L}}\right)^{2}\right\} \\
& \quad \times\left\{\left(E_{\mathrm{L}}+E_{\mathrm{H}}+m_{\mathrm{L}}+m_{\mathrm{H}}\right)^{2}\right. \\
& \left.\left.\quad \times\left(E_{\mathrm{L}}+m_{\mathrm{L}}\right)\left(E_{\mathrm{H}}+m_{\mathrm{H}}\right)\right\}^{-1}\right] .
\end{aligned}
$$

Here $m_{\mathrm{L}}$ and $m_{\mathrm{H}}$ are the masses of the light particle and the heavy fragment, respectively. To convert the two particle cross section into the measured two particle distribution function $\mathrm{d} N / \mathrm{d} E_{\mathrm{Hm}} \mathrm{d} \Omega_{\mathrm{Hm}} \mathrm{d} E_{\mathrm{Lm}} \mathrm{d} \Omega_{\mathrm{Lm}}$ at the measured laboratory angles, $\Omega_{\mathrm{Hm}}$ and $\Omega_{\mathrm{Lm}}$, and the deduced laboratory energies, $E_{\mathrm{Hm}}$ and $E_{\mathrm{L}, \mathrm{m}}$, which include a correction for energy loss in target and detector foll, one must consider the distribution of interaction points, $h\left(\boldsymbol{r}_{\mathrm{tgt}}\right)$, in the target beam spot which 
causes the actual emission angles $\Omega_{\mathrm{L}}$ and $\Omega_{\mathrm{H}}$ to differ from the values $\Omega_{\mathrm{Lc}}$ and $\Omega_{\mathrm{Hc}}$, deduced by assuming the reaction to occur at the center of the target. In addition, one must account for the difference between the corrected energies, $\tilde{E}_{\mathrm{L}}$ and $\tilde{E}_{\mathrm{H}}$, of the particles after the entrance foil of the detector telescopes (calculated by assuming the reaction to occur at the center of the target) and the original energies, $E_{\mathrm{L}}$ and $E_{\mathrm{H}}$, inside the target. These differences are represented by the distributions, $\Delta_{\mathrm{L}}\left(E_{\mathrm{L}}, \tilde{E}_{\mathrm{L}}\right)$ and $\Delta_{\mathrm{H}}\left(E_{\mathrm{H}}, \tilde{E}_{\mathrm{H}}\right)$, of energy losses (1ncluding energy loss straggling) in the target and entrance foil of the telescope. Likewise, the angles, $\tilde{\Omega}_{L}$ and $\tilde{\Omega}_{\mathrm{H}}$, of the particles at the entrance foil of the detector telescopes differ from the angles inside the target according to the distributions, $\delta_{\mathrm{L}}\left(\Omega_{\mathrm{Lc}}, \tilde{\Omega}_{\mathrm{L}}\right)$ and $\delta_{\mathrm{H}}\left(\Omega_{\mathrm{Hc}}, \tilde{\Omega}_{\mathrm{H}}\right)$, for multiple scattering in the target. Finally, one must consider the detector angular resolution functions, $A_{\mathrm{H}}\left(\tilde{\Omega}_{\mathrm{H}}, \Omega_{\mathrm{Hm}}, \tilde{E}_{\mathrm{H}}\right)$ and $A_{\mathrm{L}}\left(\tilde{\Omega}_{\mathrm{L}}, \Omega_{\mathrm{Lm}}\right.$, $\tilde{E}_{\mathrm{L}}$ ) (which include the effects of multiple scattering in the entrance foil of the telescope and the energy dependence of the position resolution), the detector energy resolution functions, $R_{\mathrm{H}}\left(E_{\mathrm{H}}, E_{\mathrm{Hm}}\right)$ and $R_{\mathrm{L}}\left(E_{\mathrm{L}}, E_{\mathrm{Lm}}\right)$, and the detector efficiencies $D_{\mathrm{L}}\left(\tilde{E}_{\mathrm{L}}, \tilde{\Omega}_{\mathrm{L}}\right)$ and $D_{\mathrm{H}}\left(\tilde{E}_{\mathrm{H}}\right.$, $\tilde{\Omega}_{H}$ ) which account for the loss of efficiency in the telescope due to multiple scattering in the telescope stack. In terms of these quantities, one obtains

$$
\begin{aligned}
& \frac{\mathrm{d} E_{\mathrm{Hm}} \frac{\mathrm{d} N}{\mathrm{~d} \Omega_{H m}} \mathrm{~d} E_{\mathrm{Lm}} \mathrm{d} \Omega_{L m}}{=} Q N_{\mathrm{tg}} \int \mathrm{d}^{2} r_{\mathrm{lg}} \mathrm{d} E_{\mathrm{H}} \mathrm{d} E_{\mathrm{L}} \mathrm{d} \Omega_{\mathrm{Hc}} \mathrm{d} \Omega_{\mathrm{Lc}} \mathrm{d} \tilde{E}_{\mathrm{H}} \mathrm{d} \tilde{E}_{\mathrm{L}} \mathrm{d} \tilde{\Omega}_{\mathrm{H}} \mathrm{d} \tilde{\Omega}_{\mathrm{L}} \\
& \quad \times D_{\mathrm{H}}\left(\tilde{E}_{\mathrm{H}}, \tilde{\Omega}_{L}\right) D_{\mathrm{L}}\left(\tilde{E}_{\mathrm{L}}, \tilde{\Omega}_{\mathrm{L}}\right) R_{\mathrm{H}}\left(\tilde{E}_{\mathrm{H}}, E_{\mathrm{Hm}}\right) \\
& \quad \times R_{\mathrm{L}}\left(\tilde{E}_{\mathrm{L}}, E_{\mathrm{LM}}\right) A_{\mathrm{H}}\left(\tilde{\Omega}_{\mathrm{H}}, \Omega_{\mathrm{Hm}}, \tilde{E}_{\mathrm{H}}\right) A_{\mathrm{L}}\left(\tilde{\Omega}_{\mathrm{L}}, \Omega_{\mathrm{Lm}}, \tilde{E}_{\mathrm{L}}\right) \\
& \quad \times \Delta_{\mathrm{H}}\left(E_{\mathrm{H}}, \tilde{E}_{\mathrm{H}}\right) \Delta_{\mathrm{L}}\left(E_{\mathrm{L}}, \tilde{E}_{\mathrm{L}}\right) \delta_{\mathrm{H}}\left(\Omega_{\mathrm{Hc}}, \tilde{\Omega}_{\mathrm{H}}\right) \\
& \quad \times \delta_{\mathrm{L}}\left(\Omega_{\mathrm{Lc}}, \tilde{\Omega}_{\mathrm{L}}\right) \tilde{J}\left(\Omega_{\mathrm{H}}, \Omega_{\mathrm{Hc}}\right) \tilde{J}\left(\Omega_{\mathrm{L}}, \Omega_{\mathrm{Lc}}\right) \\
& \quad \times h\left(\boldsymbol{r}_{\mathrm{gt}}\right) \frac{\mathrm{d} \sigma\left(E_{\mathrm{H}}, \Omega_{\mathrm{H}}, E_{\mathrm{L}}, \Omega_{\mathrm{L}}\right)}{\mathrm{d} E_{\mathrm{H}} \mathrm{d} \Omega_{\mathrm{H}} \mathrm{d} E_{\mathrm{L}} \mathrm{d} \Omega_{\mathrm{L}}}
\end{aligned}
$$

where $Q$ is the number of beam particles which traverse the target during the experiment, $N_{\mathrm{tg}}$ is the number of target nuclei per unit area and $\tilde{J}\left(\Omega_{\mathrm{H}}, \Omega_{\mathrm{Hc}}\right)$ and $\tilde{J}\left(\Omega_{\mathrm{L}}\right.$, $\Omega_{\mathrm{Lc}}$ ) are the Jacobians of the transformation from the spatial coordinate system centered at point of interaction in the target and the coordinate system whose origin is at the center of the target.

To obtain the yield $Y_{c}\left(E_{\text {mea }}^{*}\right)$ experimentally, one bins the data with respect to the measured energies and angles, calculates the mean excitation energy $E_{\text {mea }}^{*}$ corresponding the energies and angles of these bins and stores the data in the correct element of the array
$Y_{c}\left(E_{\text {mea }}^{*}\right)$ corresponding to the calculated value of $E_{\text {mea }}$. We designate this operation as

$$
\begin{aligned}
Y_{c}\left(E_{\text {mea }}^{*}\right)= & \sum_{\left(E_{\mathrm{t}}^{*}=E_{\mathrm{med}}^{*}\right)} \int_{\mathrm{bln}_{i}} \mathrm{~d} E_{\mathrm{Hm}} \mathrm{d} E_{\mathrm{Lm}} \mathrm{d} \Omega_{\mathrm{Hm}} \mathrm{d} \Omega_{\mathrm{Lm}} \\
& \times \frac{\mathrm{d} N}{\mathrm{~d} E_{\mathrm{Hm}} \mathrm{d} \Omega_{\mathrm{Hm}} \mathrm{d} E_{\mathrm{Lm}} \mathrm{d} \Omega_{\mathrm{Lm}}} .
\end{aligned}
$$

Finally, to simplify the calculation further, we approximate eq. (9) by

$$
\begin{aligned}
Y_{c}\left(E_{\text {mea }}^{*}\right)= & \left.\sum_{J} \Delta \frac{\mathrm{d} n\left(E^{*}\right)}{\mathrm{d} E^{*}}\right|_{E^{*}=E_{j}^{*}} \\
& \times \frac{1}{\Delta} \int_{E_{j}^{*}-\Delta / 2}^{E_{j}^{*}+\Delta / 2} \mathrm{~d} E^{*} \epsilon\left(E^{*}, E_{\text {mea }}^{*}\right) \\
= & \left.\sum_{j} \Delta \frac{\mathrm{d} n\left(E^{*}\right)}{\mathrm{d} E^{*}}\right|_{E^{*}=E_{j}^{*}} \bar{\epsilon}\left(E_{J}^{*}, E_{\text {mea }}^{*}\right) .
\end{aligned}
$$

Here $E_{j+1}^{*}-E_{J}^{*}=\Delta$, and $\Delta$ is chosen sufficiently small that this approximation is accurate. Then, the averaged efficiency $\bar{\epsilon}$ becomes

$$
\begin{aligned}
& \bar{\epsilon}\left(E_{,}^{*}, E_{\text {mea }}^{*}\right) \\
& =Q N_{\mathrm{lgt}} \sum_{\left(E_{i}^{*}=E_{\mathrm{med}}^{*}\right)} \int_{\text {bin, }} \mathrm{d} E_{\mathrm{Hm}} \mathrm{d} E_{\mathrm{Lm}} \mathrm{d} \Omega_{\mathrm{Hm}} \mathrm{d} \Omega_{\mathrm{Lm}} \frac{1}{\Delta} \\
& \times \int_{E_{i}^{*}-\Delta / 2}^{E_{1}^{*}+\Delta / 2} \mathrm{~d} E^{*} \\
& \times\left\{\int \mathrm{d}^{2} \boldsymbol{r}_{\mathrm{igt}} \mathrm{d} E_{\mathrm{H}} \mathrm{d} E_{\mathrm{L}} \mathrm{d} \Omega_{\mathrm{HC}} \mathrm{d} \Omega_{\mathrm{Lc}} \mathrm{d} \tilde{E}_{\mathrm{H}} \mathrm{d} \tilde{E}_{\mathrm{L}} \mathrm{d} \tilde{\Omega}_{\mathrm{H}} \mathrm{d} \tilde{\Omega}_{\mathrm{L}}\right. \\
& \times D_{\mathrm{H}}\left(\tilde{E}_{\mathrm{H}}, \tilde{\Omega}_{\mathrm{H}}\right) D_{\mathrm{L}}\left(\tilde{E}_{\mathrm{L}}, \tilde{\Omega}_{\mathrm{L}}\right) R_{\mathrm{H}}\left(\tilde{E}_{\mathrm{H}}, E_{\mathrm{Hm}}\right) \\
& \times R_{\mathrm{L}}\left(\tilde{E}_{\mathrm{L}}, E_{\mathrm{Lm}}\right) A_{\mathrm{H}}\left(\tilde{\Omega}_{\mathrm{H}}, \Omega_{\mathrm{Hm}}, \tilde{E}_{\mathrm{H}}\right) A_{\mathrm{L}}\left(\tilde{\Omega}_{\mathrm{L}}, \Omega_{\mathrm{Lm}}, \tilde{E}_{\mathrm{L}}\right) \\
& \times \Delta_{\mathrm{H}}\left(E_{\mathrm{H}}, \tilde{E}_{\mathrm{H}}\right) \Delta_{\mathrm{L}}\left(E_{\mathrm{L}}, \tilde{E}_{\mathrm{L}}\right) \delta_{\mathrm{H}}\left(\Omega_{\mathrm{H} c}, \tilde{\Omega}_{\mathrm{H}}\right) \\
& \times \delta_{\mathrm{L}}\left(\Omega_{\mathrm{Lc}}, \tilde{\Omega}_{\mathrm{L}}\right) \tilde{J}\left(\Omega_{\mathrm{H}}, \Omega_{\mathrm{Hc}}\right) \tilde{J}\left(\Omega_{\mathrm{L}}, \Omega_{\mathrm{Lc}}\right) \\
& \left.\times h\left(r_{\mathrm{tgt}}\right) \frac{\partial\left(E_{\mathrm{rel}}, \Omega_{\mathrm{cm}}, E_{\mathrm{tol}}, \Omega_{\mathrm{tot}}\right)}{\partial\left(E_{\mathrm{H}}, \Omega_{\mathrm{H}}, E_{\mathrm{L}}, \Omega_{\mathrm{L}}\right)} \frac{\mathrm{d} \sigma\left(E_{\mathrm{tot}}, \Omega_{\mathrm{tot}}\right)}{\mathrm{d} E_{\mathrm{tot}} \mathrm{d} \Omega_{\mathrm{tot}}} \frac{1}{4 \pi}\right\} .
\end{aligned}
$$

This expression is relatively straightforward to evaluate. For the efficiency calculations given here, the integration over $E^{*}, r_{\mathrm{tgt}}, E_{\mathrm{H}}, E_{\mathrm{L}}, \Omega_{\mathrm{Hc}}, \Omega_{\mathrm{Lc}}, \tilde{E}_{\mathrm{H}}, \tilde{E}_{\mathrm{L}}$, $\tilde{\Omega}_{\mathrm{H}}, \quad \tilde{\Omega}_{\mathrm{L}}$ are performed by a Monte Carlo sampling algorithm. The cross section, $\mathrm{d} \sigma\left(E_{\mathrm{tot}}, \Omega_{\mathrm{tot}}\right) / \mathrm{d} E_{\mathrm{tot}} \mathrm{d} \Omega_{\mathrm{tot}}$, used in eq. (17), was determined by fitting the inclusive data for particle stable nuclei of the same mass. The detector resolution and dependence of this resolution on the particle energy and mass were determined from the experimental and calibration runs.

Since no time is spent calculating the trajectories of particles which pass between detectors, direct integration proves to be considerably more efficient than Monte Carlo event simulation for calculatıng the efficiency. Direct comparisons between the two techniques have 
been made using calculations for an 18 element hodoscope [14] used in measurements of ${ }^{16} \mathrm{O}$ induced reactions on ${ }^{197} \mathrm{Au}$ at $E / A=94 \mathrm{MeV}$. The Monte Carlo event simulation was performed with the simulation program of ref. [2]. To better than 1\% accuracy the present efficiency calculation agrees with calculations using the event simulation program. For this case, however, direct integration is about a factor of 20 faster than Monte Carlo event simulation.

For determination of the background yield, $Y_{\text {back }}\left(E_{\text {mea }}\right)$, it is necessary to perform an identical event binning for the product, $\sigma_{L} \sigma_{H}$, of single cross sections (see eq. (13)) as was performed in eq. (17) for the coincidence yield. For position sensitive detectors, it is considerably easier to fit the singles cross sections $\sigma_{\mathrm{L}}$ and $\sigma_{H}$ with a moving source parametrization and integrate the parameterized cross sections than to perform a mixed single-particle event analysis [15]. Since the excitation energy $E^{*}$ is rather trivially related to the relative momentum $\Delta p$, it is equivalent and actually easier to define a correlation function $\left[1+\tilde{R}_{\text {back }}\left(E^{*}\right)\right]$ $=\left[1+R_{\text {back }}(\Delta p)\right]$, which satisfies the equation

$Y_{\text {back }}\left(p_{\mathrm{H}}, p_{\mathrm{L}}\right)=C \sigma_{\mathrm{L}}\left(p_{\mathrm{L}}\right) \sigma_{\mathrm{H}}\left(p_{\mathrm{H}}\right)\left(1+\tilde{R}_{\text {back }}\left(E^{*}\right)\right)$.

Summing both sides of equation for a fixed excitation energy $E_{\text {mea }}^{*}$ provides

$$
\begin{aligned}
& Y_{\text {back }}\left(E_{\text {mea }}^{*}\right) \\
& =C\left[1+\tilde{R}_{\text {back }}\left(E_{\text {mea }}^{*}\right)\right] \\
& \times \sum_{\left(E_{i}^{*}=E_{\text {mea }}^{*}\right)} \int_{\mathrm{bln},} \mathrm{d} E_{\mathrm{Hm}} \mathrm{d} E_{\mathrm{Lm}} \mathrm{d} \Omega_{\mathrm{Hm}} \mathrm{d} \Omega_{\mathrm{Lm}} \\
& \times \frac{1}{\Delta} \int_{E_{j}^{*}-\Delta / 2}^{E_{j}^{*}+\Delta / 2} \mathrm{~d} E^{*} \\
& \times\left\{\int \mathrm{d}^{2} \boldsymbol{r}_{\mathrm{tgt}} \mathrm{d} E_{\mathrm{H}} \mathrm{d} E_{\mathrm{L}} \mathrm{d} \Omega_{\mathrm{Hc}} \mathrm{d} \Omega_{\mathrm{Lc}} \mathrm{d} \tilde{E}_{\mathrm{H}} \mathrm{d} \tilde{E}_{\mathrm{L}} \mathrm{d} \tilde{\Omega}_{\mathrm{H}} \mathrm{d} \tilde{\Omega}_{\mathrm{L}}\right. \\
& \times D_{\mathrm{H}}\left(\tilde{E}_{\mathrm{H}}, \tilde{\Omega}_{\mathrm{H}}\right) D_{\mathrm{L}}\left(\tilde{E}_{\mathrm{L}}, \tilde{\Omega}_{\mathrm{L}}\right) R_{\mathrm{H}}\left(\tilde{E}_{\mathrm{H}}, E_{\mathrm{Hm}}\right) \\
& \times R_{\mathrm{L}}\left(\tilde{E}_{\mathrm{L}}, E_{\mathrm{Lm}}\right) A_{H}\left(\tilde{\Omega}_{\mathrm{H}}, \Omega_{\mathrm{Hm}}, \tilde{E}_{\mathrm{H}}\right) A_{\mathrm{L}}\left(\tilde{\Omega}_{\mathrm{L}}, \Omega_{\mathrm{Lm}}, \tilde{E}_{\mathrm{H}}\right) \\
& \times \Delta_{\mathrm{H}}\left(E_{\mathrm{H}}, \tilde{E}_{\mathrm{H}}\right) \Delta_{\mathrm{L}}\left(E_{\mathrm{L}}, \tilde{E}_{\mathrm{L}}\right) \delta_{\mathrm{h}}\left(\Omega_{\mathrm{Hc}}, \tilde{\Omega}_{\mathrm{H}}\right) \\
& \times \delta_{\mathrm{L}}\left(\Omega_{\mathrm{Lc}}, \tilde{\Omega}_{\mathrm{L}}\right) \tilde{J}\left(\Omega_{\mathrm{H}}, \Omega_{\mathrm{Hc}}\right) \tilde{J}\left(\Omega_{\mathrm{L}}, \Omega_{\mathrm{Lc}}\right) \\
& \left.\times h\left(r_{\mathrm{tgl}}\right) \frac{\mathrm{d} \sigma_{\mathrm{H}}\left(E_{\mathrm{H}}, \Omega_{\mathrm{H}}\right)}{\mathrm{d} E_{\mathrm{H}} \mathrm{d} \Omega_{\mathrm{H}}} \frac{\mathrm{d} \sigma_{\mathrm{L}}\left(E_{\mathrm{L}}, \Omega_{\mathrm{L}}\right)}{\mathrm{d} E_{\mathrm{L}} \mathrm{d} \Omega_{\mathrm{L}}}\right\} \text {. }
\end{aligned}
$$

Away from the peaks corresponding to the decay of excited states, where $Y_{c}$ is small,

$$
\frac{Y_{\text {tot }}\left(E_{\text {mea }}^{*}\right)}{Y_{\text {back }}\left(E_{\text {mea }}^{*}\right)}=1 \text {. }
$$

Using this relationship $\left[1+\tilde{R}_{\text {back }}\left(E_{\text {mea }}^{*}\right)\right]$ is determined empirically.

\section{Acknowledgements}

We would like to acknowledge many helpful discussıons with Dennis Swan and John Yurkon. This work was supported by the US National Science Foundation under Grant No. PHY 86-11210. W.G.L. acknowledges the receipt of a US Presidential Young Investigator Award and equipment grant from EG\&G Ortec.

\section{References}

[1] D.J. Morrissey, W. Benenson, E. Kashy, B. Sherrill, A.D. Panagiotou, R.A. Blue, R.M. Ronningen, J. van der Plicht and H. Utsunomiya, Phys. Lett. B148 (1984) 423.

[2] J. Pochodzalla, C.K. Gelbke, W.G. Lynch, M. Marer, D. Ardouin, H. Delagrange, H. Doubre, C. Grégoire, A. Kyanowski, W. Mittig, A. Péghaire, J. Péter, F. SantLaurent, B. Zwieglinskı, G. Blizard, F. Lefèbvres, B. Tamain, J. Québert, Y.P. Viyogi, W.A. Friedman and D.H. Boal, Phys. Rev. C35 (1987) 1695.

[3] Z. Chen, C.K. Gelbke, J. Pochodzalla, C.B. Chitwood, D.J. Fields, W.G. Gong, W.G. Lynch and M.B. Tsang, Nucl. Phys. A473 (1987) 564.

[4] For examples of models where eq. (1) should be appropriate see J. Randrup and S.E. Koonın, Nucl. Phys. A356 (1981) 223; W.A. Friedman and W.G. Lynch, Phys. Rev. C28 (1983) 16.

[5] J.T. Walton, H.A. Sommer, D.E. Greıner and F.S. Bieser, IEEE Trans. Nucl. Sci. NS-25 (1978) 391.

[6] V. Radeka, IEEE Trans. Nucl. Sci. NS-21 (1974) 51.

[7] F. Sauli, Lectures given in the Academic Training Programme of CERN, CERN 77-09 (1977).

[8] J. Va'vra, Nucl. Instr. and Meth. A252 (1986) 547

[9] F.S. Goulding and B.G. Harvey, Ann. Rev. Nucl. Sc1. 25 (1975) 167.

[10] T. Shimoda, M. Ishihara, K. Nagatanı and T. Nomura, Nucl. Instr. and Meth. 165 (1979) 261.

[11] J.F. Ziegler, Stopping Cross-sections for Energetic Ions in All Elements, vol. 5 (Pergamon, New York, 1980).

[12] F. Ajzenberg-Selove, Nucl. Phys. A449 (1986) 1.

[13] T. Murakami et al,, to be published.

[14] Z. Chen, C.K. Gelbke, W.G. Gong, Y.D. K.m, W.G. Lynch, M.R. Marer, J. Pochodzalla, M.B. Tsang, F. SaintLaurent, D. Ardouin, H. Delagrange, H. Doubre, J. Kasagı, A. Kyanowski, A. Péghaire, J. Péter, E. Rosato, G. Blizard, F. Lefèbvres, B. Tamain, J. Québert and Y.P. Viyogi, Phys. Rev, C36 (1987) 2297.

[15] F. Zarbakhsh, A.L. Sagle, F. Brochard, T.A. Mulera, V. Perez-Mendez, R. Talaga, I. Tanihata, J.B. Carroll, K.S. Ganezer, G. Igo, J. Oostens, D. Woodard and R. Sutter, Phys. Rev. Lett. 46 (1981) 1268. 